\title{
Model of dissipative dielectric elastomers
}

\section{Citation}

Chiang Foo, Choon, Shengqiang Cai, Soo Jin Adrian Koh, Siegfried Bauer, and Zhigang Suo. 2012. “Model of Dissipative Dielectric Elastomers." Journal of Applied Physics 111 (3): 34102. https:// doi.org/10.1063/1.3680878.

\section{Permanent link}

http://nrs.harvard.edu/urn-3:HUL.InstRepos:41461227

\section{Terms of Use}

This article was downloaded from Harvard University's DASH repository, and is made available under the terms and conditions applicable to Other Posted Material, as set forth at http:// nrs.harvard.edu/urn-3:HUL.InstRepos:dash.current.terms-of-use\#LAA

\section{Share Your Story}

The Harvard community has made this article openly available.

Please share how this access benefits you. Submit a story.

\section{Accessibility}




\title{
Model of dissipative dielectric elastomers
}

\author{
Choon Chiang Foo, ${ }^{1,2}$ Shengqiang Cai, ${ }^{1}$ Soo Jin Adrian Koh, ${ }^{2,3}$ Siegfried Bauer, ${ }^{4}$ \\ and Zhigang Suo ${ }^{1, a)}$ \\ ${ }^{1}$ School of Engineering and Applied Sciences, Kavli Institute for Nanobio Science and Technology, \\ Harvard University, Cambridge, Massachusetts 02138, USA \\ ${ }^{2}$ Institute of High Performance Computing, 1 Fusionopolis Way, \#16-16 Connexis, Singapore 138632, \\ Singapore \\ ${ }^{3}$ Engineering Science Programme and Department of Civil and Environmental Engineering, National \\ University of Singapore, Kent Ridge, Singapore 119260, Singapore \\ ${ }^{4}$ Soft-Matter Physics Department, Johannes Kepler University Linz, A-4040 Linz, Austria
}

(Received 17 October 2011; accepted 17 December 2011; published online 3 February 2012)

\begin{abstract}
The dynamic performance of dielectric elastomer transducers and their capability of electromechanical energy conversion are affected by dissipative processes, such as viscoelasticity, dielectric relaxation, and current leakage. This paper describes a method to construct a model of dissipative dielectric elastomers on the basis of nonequilibrium thermodynamics. We characterize the state of the dielectric elastomer with kinematic variables through which external loads do work, and internal variables that measure the progress of the dissipative processes. The method is illustrated with examples motivated by existing experiments of polyacrylate very-high-bond dielectric elastomers. This model predicts the dynamic response of the dielectric elastomer and the leakage current behavior. We show that current leakage can be significant under large deformation and for long durations. Furthermore, current leakage can result in significant hysteresis for dielectric elastomers under cyclic voltage. (C) 2012 American Institute of Physics. [doi:10.1063/1.3680878]
\end{abstract}

\section{INTRODUCTION}

A dielectric elastomer transducer consists of a thin membrane of the elastomer, sandwiched between two compliant electrodes (Fig. 1(a)). The electrodes are connected to a power source through a conducting wire. Subject to a voltage, the electric current in the conducting wire partly leaks through the membrane, and partly adds charges to the electrodes (Fig. 1(b)). The opposite charges on the electrodes polarize the dielectric. Attractive electrostatic forces between the opposite charges reduce thickness and expand area of the membrane-the dielectric elastomer performs as an actuator. ${ }^{1,2}$ On the other hand, when a pre-stretched and precharged membrane is mechanically relaxed in the open-circuit condition, the membrane boosts the voltage between the electrodes - the dielectric elastomer performs as a generator.

As a result of their fast response time and high energy density, dielectric elastomers (DEs) have been developed as artificial muscles, Braille displays, life-like robots, tunable lens, and power generators. ${ }^{3-9}$ To perform as an actuator and a generator, a dielectric elastomer is often subject to transient, time-dependent forces and voltages. ${ }^{9-13}$ The performance will therefore be affected by mechanisms of dissipation in the system. ${ }^{13-15}$ In particular, experiments have shown that viscoelasticity and current leakage can adversely affect the performance of a transducer and limit its application. ${ }^{9,11-17}$ This paper presents a model that incorporates the major dissipative mechanisms in dielectric elastomers, so as to predict how these dissipative processes affect the performance of dielectric elastomer transducers.

\footnotetext{
a) Author to whom correspondence should be addressed. Electronic mail: suo@seas.harvard.edu.
}

The state of equilibrium of a dielectric elastomer transducer is described by four types of variables: force, displacement, voltage and charge. One may choose to vary any two of the four variables independently, with the other two variables determined by the material properties. Therefore, the transducer is an electromechanical system with two degrees of freedom. For instance, one may choose to vary the force and voltage as inputs to the transducer. Subject to a mechanical force and voltage, the transducer takes time to equilibrate to its state of kinematic deformation and charge polarization. This process is known as relaxation.

Subject to a mechanical force, a dielectric elastomer relaxes to a new state of deformation over a characteristic time, known as the viscoelastic relaxation time, $\tau_{V}$. An elastomer consists of a network of polymer chains (Fig. 2(a)). Each polymer chain is connected to other polymer chains at specific points, by covalent crosslinks, forming a network. A sufficiently flexible polymer chain may twist and coil, and entangle with other chains. When a force is applied to the elastomer, localized slipping and sliding of polymer chains occur. Viscoelastic relaxation is a consequence of entangled polymer chains responding to a force. Experiments have shown that for polyacrylate very-high-bond (VHB) elastomer, which is a commercial tape from $3 \mathrm{M}, \tau_{V}$ is on the order of $10^{2}$ seconds at room temperature. ${ }^{16,18-20}$

Subject to a voltage, the dielectric elastomer relaxes to a new state of polarization over a characteristic time, known as the dielectric relaxation time, $\tau_{d}$. Dielectric relaxation is the process of re-orientation of molecular dipoles in a polar dielectric (Fig. 2(b)). The re-alignment is on the molecular scale. The dielectric relaxation time $\tau_{d}$ strongly depends on temperature, especially in the vicinity of the glass transition. On the basis of experiments on polymer dielectrics, ${ }^{21,22}$ we 
Reference state
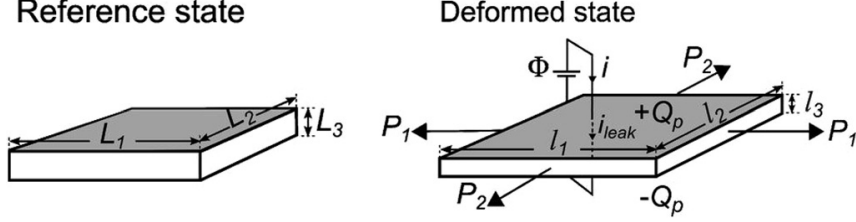

FIG. 1. In the reference state, a membrane of a dielectric elastomer is undeformed, and has dimensions $L_{1}, L_{2}$, and $L_{3}$. In the deformed state, subject to forces $P_{1}$ and $P_{2}$, as well as voltage $\Phi$, the membrane attains dimensions $l_{l}$, $l_{2}$, and $l_{3}$. The elastomer is viscoelastic and is an imperfect insulator. The current $i$ in the conducting wire partly charges the electrodes, and partly leaks through the membrane.

assume that $\tau_{d}$ is on the order of $10^{-6}$ seconds at room temperature for dielectric elastomers in the rubbery state.

The relaxation processes mentioned above involve localized translation and rotation of single molecules, a group of molecules, or molecular chains. We next describe a nonequilibrium process that involves long-range transport through the dielectric (Fig. 3). When a dielectric is connected to a power source, a small current may flow through the dielectric. This process is known as current leakage, which is well documented in several insulator and semi-conductor systems. ${ }^{17,23-27}$ The occurrence of leakage current is a result of the transport of electrons, ions, or both, ${ }^{23-25}$ and is often linked to the presence of impurities and imperfections. ${ }^{23,24}$ In the simplest case, the leakage current density varies approximately linearly with the applied field for small electric fields $(<10 \mathrm{MV} / \mathrm{m})$, known as the Ohmic conduction.

As the electric field increases, the leakage current strongly increases; in commonly used models an exponential increase with the electric field is assumed, ${ }^{17,26}$ approaching electrical breakdown. Electrical conduction does not follow Ohm's Law (non-Ohmic). The switch from Ohmic to nonOhmic conduction is used as a precursor to indicate imminent electrical breakdown. ${ }^{15}$ Continued operation within the non-Ohmic regime may also lead to severe deterioration of the insulating properties of the dielectric. ${ }^{28-32}$

In this paper, we investigate the effects of dissipative processes on the electromechanical conversion performance of a dielectric elastomer. In particular, we develop a model that includes both local and long-range dissipation in the dielectric elastomer within a nonequilibrium thermodynamics framework. To illustrate our approach, we construct a specific model for viscoelastic dielectric elastomer and define a current leakage density based on empirical observations. In our analysis, we assume that the dielectric elastomer has a dielectric relaxation time in the order of $10^{-6} \mathrm{~s}$, and that the time scale of operation is in the order of seconds. Hence, dielectric relaxation will be ignored in our analyses. Nevertheless, our framework allows dielectric relaxation to be included for systems where the effect cannot be ignored. Finally we provide examples to show how viscoelasticity and current leakage affect the performance of a dielectric elastomer.

\section{THERMODYNAMICS OF DISSIPATIVE PROCESSES}

This section describes a thermodynamic model of dissipative dielectric elastomers. To focus on main ideas, the presentation of this section is restricted to a membrane of a dielectric elastomer undergoing homogeneous deformation. The same model is presented in a general form, in the Appendix, for a body of a dielectric elastomer undergoing inhomogeneous deformation.

Figure 1 illustrates the operation of a dielectric elastomer transducer. A membrane of a dielectric elastomer is sandwiched between two electrodes of negligible electrical resistance and mechanical stiffness compared to those of the membrane. In the reference state, the membrane is undeformed and is of dimensions $L_{1}, L_{2}$, and $L_{3}$. In the deformed state, the membrane is subject to forces $P_{1}$ and $P_{2}$, and the two electrodes connected through a conducting wire to a power source of voltage $\Phi$, while the dimensions of the membrane are $l_{1}, l_{2}$, and $l_{3}$. The forces, voltage, and dimensions are time dependent.

The charge moving in the conducting wire, $\delta Q$, partly leaks through the membrane, $\delta Q_{\text {leak }}$, and partly accumulates on the electrodes to polarize the membrane. The amount of polarizing charge on either electrode changes from $Q_{p}$ to $Q_{p}+\delta Q_{p}$. The conservation of charge requires that,

$$
\delta Q=\delta Q_{p}+\delta Q_{\text {leak }}
$$

We shall restrict our analysis to isothermal processes. Let the Helmholtz free energy of the membrane be $F$. Associated with small changes in the dimensions of the membrane, $\delta l_{1}$, $\delta l_{2}$, and $\delta l_{3}$, the forces do work $P_{1} \delta l_{1}+P_{2} \delta l_{2}$. Associated with a small amount of charge moving through the conducting wire, $\delta Q$, the voltage does work $\Phi \delta Q$. Thermodynamics (a)

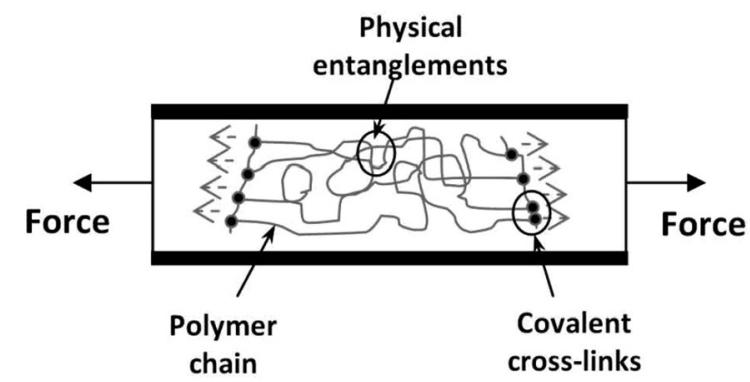

(b)

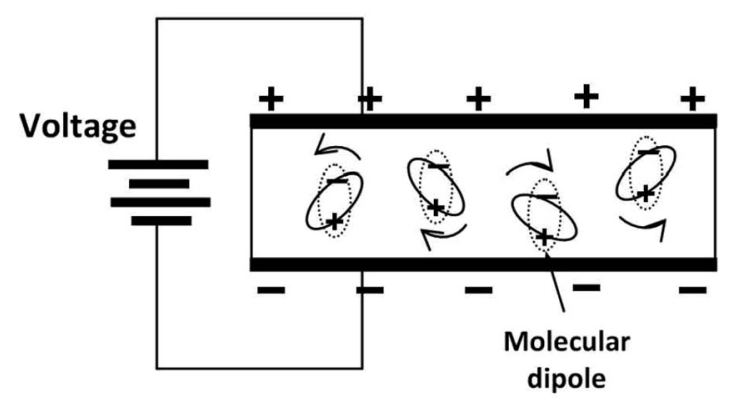

FIG. 2. Schematics of local dissipative processes: (a) viscoelastic relaxation due to sliding of polymer chains; (b) dielectric relaxation due to re-orientation of dipoles along the direction of the applied electric field. 
(a)

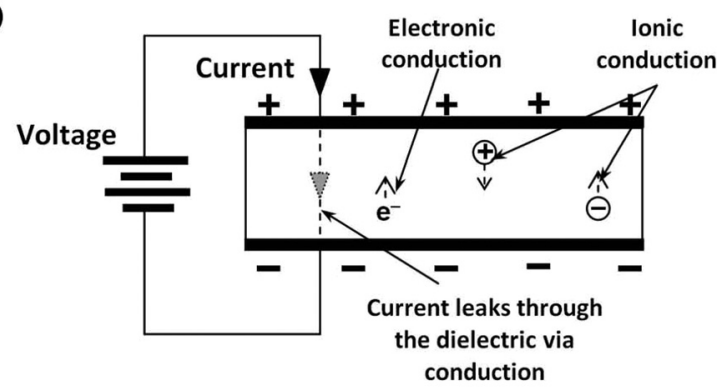

(b)

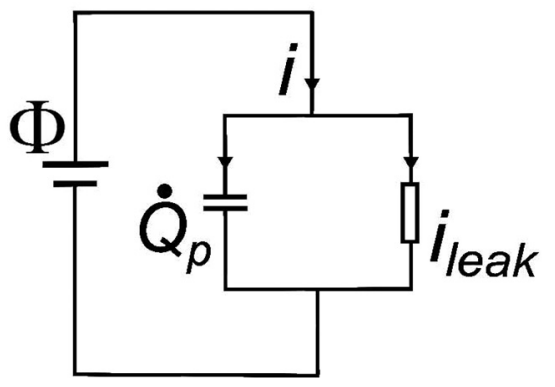

FIG. 3. (a) Schematics of current leakage through the dielectric: charge transport is due to the movement of electrons $\left(\mathrm{e}^{-}\right)$and ions $(+/-)$under an applied electric field. (b) To model current leakage, the dielectric elastomer is considered as a capacitor with a resistor in parallel. By the principle of charge conservation, the current consumed by the battery $i$ is the sum of the charge flow rate to the capacitor $\dot{Q}_{p}$ and the current leakage through the resistor $i_{\text {leak }}$.

dictates that the increase in the free energy should not exceed the combined work done by the forces and voltage,

$$
\delta F \leq P_{1} \delta l_{1}+P_{2} \delta l_{2}+\Phi \delta Q .
$$

The equality holds when the membrane undergoes quasiequilibrium processes. The inequality holds when the membrane undergoes nonequilibrium processes. For the inequality to be meaningful, the small changes are time directed: $\delta f$ means the change of the quantity $f$ from one time to a slightly later time.

The thermodynamic inequality (2) should hold for every dissipative process in the membrane. As discussed before, the dissipative processes in the membrane may be classified into two types. The leakage current involves long-range transport of charges, while viscoelasticity and dielectric relaxation involve local molecular movements. The two types of processes are separately dissipative. Thus, thermodynamics requires that,

$$
\Phi \delta Q_{\text {leak }} \geq 0 .
$$

This inequality is readily understood: the charge leaks through the membrane in the direction from the highpotential electrode to the low-potential electrode. Furthermore, viscoelastic and dielectric relaxation are dissipative, so that,

$$
\delta F \leq P_{1} \delta l_{1}+P_{2} \delta l_{2}+\Phi \delta Q_{p} .
$$

The last term represents the work done by the polarizing charge on the electrodes.
The density of the Helmholtz free energy is defined as $W=F /\left(L_{1} L_{2} L_{3}\right)$. The stretches of the elastomer in the three directions are defined by $\lambda_{1}=l_{1} / L_{1}, \lambda_{2}=l_{2} / L_{2}$, and $\lambda_{3}=l_{3} / L_{3}$. The stresses are defined by $\sigma_{1}=P_{1} /\left(l_{2} l_{3}\right)$ and $\sigma_{2}=P_{2} /\left(l_{1} l_{3}\right)$. The electric field is $E=\Phi / l_{3}$, and the electric displacement is defined by $D=Q_{p} /\left(l_{1} l_{2}\right)$. By definition, the amount of polarizing charge accumulated on either electrode is $Q_{p}=D l_{1} l_{2}$. Associated with small changes in the dimensions of the membrane and the electric displacement, the polarizing charge changes by $\delta Q_{p}=D l_{1} \delta l_{2}+D l_{2} \delta l_{1}$ $+l_{1} l_{2} \delta D$. Inserting this expression into (4), and dividing the resulting inequality by the volume of the undeformed membrane, $L_{1} L_{2} L_{3}$, we obtain that,

$$
\begin{aligned}
\delta W \leq & \left(\sigma_{1}+E D\right) \lambda_{2} \lambda_{3} \delta \lambda_{1}+\left(\sigma_{2}+E D\right) \lambda_{1} \lambda_{3} \delta \lambda_{2} \\
& +\lambda_{1} \lambda_{2} \lambda_{3} E \delta D .
\end{aligned}
$$

The elastomer is assumed to be incompressible, so that $\lambda_{1} \lambda_{2} \lambda_{3}=1$. We will use $\lambda_{1}$ and $\lambda_{2}$ as independent variables, and calculate $\lambda_{3}$ from $\lambda_{3}=\left(\lambda_{1} \lambda_{2}\right)^{-1}$. The density of the Helmholtz free energy is assumed to be a function of a set of independent variables,

$$
W=W\left(\lambda_{1}, \lambda_{2}, D, \xi_{\alpha}, \xi_{\beta}, \ldots\right) .
$$

Here $\xi_{\alpha}, \xi_{\beta}, \ldots$ represent a set of internal variables that characterize the progress of dissipative processes in the membrane. In particular, $\xi_{\alpha}, \xi_{\beta}, \ldots$ can represent the progress of viscoelastic and dielectric relaxation. Associated with small changes in the independent variables, the density of the Helmholtz free energy changes by,

$$
\delta W=\frac{\partial W}{\partial \lambda_{1}} \delta \lambda_{1}+\frac{\partial W}{\partial \lambda_{2}} \delta \lambda_{2}+\frac{\partial W}{\partial D} \delta D+\sum_{\gamma} \frac{\partial W}{\partial \xi_{\gamma}} \delta \xi_{\gamma} .
$$

A combination of (5) and (7) gives that,

$$
\begin{aligned}
& \left(\frac{\partial W}{\partial \lambda_{1}}-\left(\sigma_{1}+E D\right) \lambda_{2} \lambda_{3}\right) \delta \lambda_{1}+\left(\frac{\partial W}{\partial \lambda_{2}}-\left(\sigma_{2}+E D\right) \lambda_{1} \lambda_{3}\right) \delta \lambda_{2} \\
& +\left(\frac{\partial W}{\partial D}-E\right) \delta D+\sum_{\gamma} \frac{\partial W}{\partial \xi_{\gamma}} \delta \xi_{\gamma} \leq 0 .
\end{aligned}
$$

This inequality can be satisfied in many ways. We adopt the following assumptions. The membrane is in mechanical and electrostatic equilibrium, so that the coefficients in front of $\delta \lambda_{1}, \delta \lambda_{2}$, and $\delta D$ vanish, giving,

$$
\begin{gathered}
\sigma_{1}+E D=\lambda_{1} \frac{\partial W\left(\lambda_{1}, \lambda_{2}, D, \xi_{\alpha}, \xi_{\beta}, \ldots\right)}{\partial \lambda_{1}}, \\
\sigma_{2}+E D=\lambda_{2} \frac{\partial W\left(\lambda_{1}, \lambda_{2}, D, \xi_{\alpha}, \xi_{\beta}, \ldots\right)}{\partial \lambda_{2}}, \\
E=\frac{\partial W\left(\lambda_{1}, \lambda_{2}, D, \xi_{\alpha}, \xi_{\beta}, \ldots\right)}{\partial D} .
\end{gathered}
$$

The membrane, however, is not in equilibrium with respect to viscoelastic and dielectric relaxation, so that,

$$
\sum_{\gamma} \frac{\partial W\left(\lambda_{1}, \lambda_{2}, D, \xi_{\alpha}, \xi_{\beta}, \ldots\right)}{\partial \xi_{\gamma}} \delta \xi_{\gamma} \leq 0 .
$$


Inequality (12) places a restriction on viscoelastic models, and its effect will become evident when a rheological model is specified.

\section{IDEAL DIELECTRIC ELASTOMERS}

Both viscoelasticity and dielectric relaxation are due to local movements of molecules, or a group of molecules (Ref. 34) (Fig. 2). Although the rotation of the dipoles may cause deformation in the elastomer, we assume that this deformation is negligible compared to that caused by stretching of the long polymer chains. For commonly used dielectrics like VHB and silicone, at room temperature and pressure, the dielectric relaxation time is orders of magnitude smaller than the viscoelastic relaxation time. ${ }^{21,22}$ Furthermore, the typical time scale of operation of dielectric elastomers is in the region of seconds, where dielectric relaxation has long taken place. Hence, we will neglect dielectric relaxation in our analyses.

An elastomer is a network of polymer chains crosslinked by covalent bonds. Each chain consists of a large number of monomers. As a result, the crosslinks have a negligible effect on the process of polarization of these monomers - that is, the elastomer polarizes nearly like a polymer melt. This picture is supported by experiments, ${ }^{35}$ which show that the permittivity of the elastomer changes insignificantly over a strain of $400 \%$. We adopt the model of ideal dielectric elastomer, ${ }^{36}$ assuming that the polarization behavior is liquid-like and independent of the state of deformation. That is, the electric displacement is taken to be linear in the electric field,

$$
D=\varepsilon E,
$$

where $\varepsilon$ is the permittivity independent of deformation.

Inserting (13) into (11) and integrating, we obtain that

$$
W\left(\lambda_{1}, \lambda_{2}, D, \xi_{\alpha}, \xi_{\beta}, \ldots\right)=W_{\text {stretch }}\left(\lambda_{1}, \lambda_{2}, \xi_{\alpha}, \xi_{\beta}, \ldots\right)+\frac{D^{2}}{2 \varepsilon} .
$$

Here $W_{\text {stretch }}$ is the Helmholtz free energy associated with the stretching of the elastomer. In the model of ideal dielectric elastomers, the stretching and polarization of the elastomer contribute to the free energy independently.

Inserting (14) into (9) and (10), we obtain that,

$$
\begin{aligned}
\sigma_{1}+\varepsilon E^{2} & =\lambda_{1} \frac{\partial W_{\text {stretch }}\left(\lambda_{1}, \lambda_{2}, \xi_{\alpha}, \xi_{\beta}, \ldots\right)}{\partial \lambda_{1}}, \\
\sigma_{2}+\varepsilon E^{2} & =\lambda_{2} \frac{\partial W_{\text {stretch }}\left(\lambda_{1}, \lambda_{2}, \xi_{\alpha}, \xi_{\beta}, \ldots\right)}{\partial \lambda_{2}} .
\end{aligned}
$$

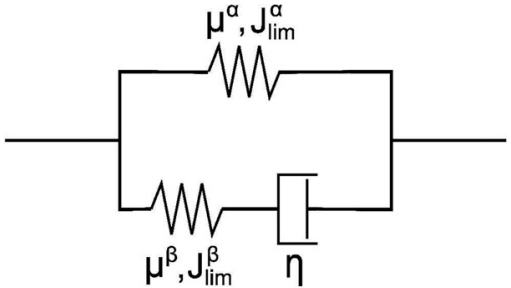

FIG. 4. Viscoelastic relaxation is modeled using two parallel units. One unit consists of spring $\alpha$, and the other unit consists of spring $\beta$ and a dashpot.

These relations confirm a widely used description of electromechanical interaction: The voltage produces a Maxwell stress of $\varepsilon E^{2}{ }^{1}$

\section{MODEL OF VISCOELASTICITY}

Viscoelastic relaxation may be represented by a rheological model of springs and dashpots. We adopt a rheological model of two parallel units: one unit consists of a spring, $\alpha$, and the other unit consists of another spring, $\beta$, and a dashpot (Fig. 4). As discussed before, the deformation of the elastomer is represented by two stretches, $\lambda_{1}$ and $\lambda_{2}$. In the rheological model, $\lambda_{1}$ and $\lambda_{2}$ are assumed to be the net stretches of both units. For spring $\alpha$, the state of deformation is characterized by stretches $\lambda_{1}$ and $\lambda_{2}$. For spring $\beta$, however, the state of deformation is characterized by different stretches, which we designate as $\lambda_{1}^{e}$ and $\lambda_{2}^{e}$. Let $\xi_{1}$ and $\xi_{2}$ be the stretches in the dashpot. We adopt the multiplication rule, ${ }^{20,37,38}$

$$
\lambda_{1}=\lambda_{1}^{e} \xi_{1}, \lambda_{2}=\lambda_{2}^{e} \xi_{2}
$$

Upon approaching a limiting stretch as a result of the finite contour length of the polymer chains, the dielectric elastomer shows pronounced stress-stiffening. To account for the effect of stress-stiffening, we represent both springs by using the Gent model. ${ }^{39}$ The free-energy function of the elastomer is the sum of the contributions from the two springs:

$$
\begin{aligned}
W_{\text {stretch }}= & -\frac{\mu^{\alpha} J_{\lim }^{\alpha}}{2} \log \left(1-\frac{\lambda_{1}^{2}+\lambda_{2}^{2}+\lambda_{1}^{-2} \lambda_{2}^{-2}-3}{J_{\lim }^{\alpha}}\right) \\
& -\frac{\mu^{\beta} J_{\lim }^{\beta}}{2} \log \left(1-\frac{\lambda_{1}^{2} \xi_{1}^{-2}+\lambda_{2}^{2} \xi_{2}^{-2}+\lambda_{1}^{-2} \lambda_{2}^{-2} \xi_{1}^{2} \xi_{2}^{2}-3}{J_{\lim }^{\beta}}\right),
\end{aligned}
$$

where $\mu^{\alpha}$ and $\mu^{\beta}$ are shear moduli of the two springs, and $J_{\lim }^{\alpha}$ and $J_{\lim }^{\beta}$ are constants related to the limiting stretches of the two springs.

Inserting (18) into (15) and (16), we obtain that,

$$
\begin{aligned}
\sigma_{1}+\varepsilon E^{2} & =\frac{\mu^{\alpha}\left(\lambda_{1}^{2}-\lambda_{1}^{-2} \lambda_{2}^{-2}\right)}{1-\left(\lambda_{1}^{2}+\lambda_{2}^{2}+\lambda_{1}^{-2} \lambda_{2}^{-2}-3\right) / J_{\lim }^{\alpha}}+\frac{\mu^{\beta}\left(\lambda_{1}^{2} \xi_{1}^{-2}-\xi_{1}^{2} \xi_{2}^{2} \lambda_{1}^{-2} \lambda_{2}^{-2}\right)}{1-\left(\lambda_{1}^{2} \xi_{1}^{-2}+\lambda_{2}^{2} \xi_{2}^{-2}+\xi_{1}^{2} \xi_{2}^{2} \lambda_{1}^{-2} \lambda_{2}^{-2}-3\right) / J_{\lim }^{\beta}}, \\
\sigma_{2}+\varepsilon E^{2} & =\frac{\mu^{\alpha}\left(\lambda_{2}^{2}-\lambda_{1}^{-2} \lambda_{2}^{-2}\right)}{1-\left(\lambda_{1}^{2}+\lambda_{2}^{2}+\lambda_{1}^{-2} \lambda_{2}^{-2}-3\right) / J_{\lim }^{\alpha}}+\frac{\mu^{\beta}\left(\lambda_{2}^{2} \xi_{2}^{-2}-\xi_{1}^{2} \xi_{2}^{2} \lambda_{1}^{-2} \lambda_{2}^{-2}\right)}{1-\left(\lambda_{1}^{2} \xi_{1}^{-2}+\lambda_{2}^{2} \xi_{2}^{-2}+\xi_{1}^{2} \xi_{2}^{2} \lambda_{1}^{-2} \lambda_{2}^{-2}-3\right) / J_{\lim }^{\beta}} .
\end{aligned}
$$


The two terms in the right-hand side of the above equations are the stresses carried by the two springs in the rheological model.

We model the dashpot as a Newtonian fluid. In the rheological model, the state of stress in the dashpot is the same as that in spring $\beta$. This state of stress is characterized by the two components given as the last term in (19) and the last term in (20). The rate of deformation in the dashpot is described by the two components $\xi_{1}^{-1} d \xi_{1} / d t$ and $\xi_{2}^{-1} d \xi_{2} / d t$. To model the dashpot as a Newtonian fluid, we relate the rate of deformation to the stress as,

$$
\begin{aligned}
& \frac{d \xi_{1}}{\xi_{1} d t}=\frac{1}{3 \eta}\left(\frac{\mu^{\beta}\left(\lambda_{1}^{2} \xi_{1}^{-2}-\xi_{1}^{2} \xi_{2}^{2} \lambda_{1}^{-2} \lambda_{2}^{-2}\right)}{1-\left(\lambda_{1}^{2} \xi_{1}^{-2}+\lambda_{2}^{2} \xi_{2}^{-2}+\xi_{1}^{2} \xi_{2}^{2} \lambda_{1}^{-2} \lambda_{2}^{-2}-3\right) / J_{\lim }^{\beta}}-\frac{\mu^{\beta}\left(\lambda_{2}^{2} \xi_{2}^{-2}-\xi_{1}^{2} \xi_{2}^{2} \lambda_{1}^{-2} \lambda_{2}^{-2}\right) / 2}{1-\left(\lambda_{1}^{2} \xi_{1}^{-2}+\lambda_{2}^{2} \xi_{2}^{-2}+\xi_{1}^{2} \xi_{2}^{2} \lambda_{1}^{-2} \lambda_{2}^{-2}-3\right) / J_{\lim }^{\beta}}\right), \\
& \frac{d \xi_{2}}{\xi_{2} d t}=\frac{1}{3 \eta}\left(\frac{\mu^{\beta}\left(\lambda_{2}^{2} \xi_{2}^{-2}-\xi_{1}^{2} \xi_{2}^{2} \lambda_{1}^{-2} \lambda_{2}^{-2}\right)}{1-\left(\lambda_{1}^{2} \xi_{1}^{-2}+\lambda_{2}^{2} \xi_{2}^{-2}+\xi_{1}^{2} \xi_{2}^{2} \lambda_{1}^{-2} \lambda_{2}^{-2}-3\right) / J_{\lim }^{\beta}}-\frac{\mu^{\beta}\left(\lambda_{1}^{2} \xi_{1}^{-2}-\xi_{1}^{2} \xi_{2}^{2} \lambda_{1}^{-2} \lambda_{2}^{-2}\right) / 2}{1-\left(\lambda_{1}^{2} \xi_{1}^{-2}+\lambda_{2}^{2} \xi_{2}^{-2}+\xi_{1}^{2} \xi_{2}^{2} \lambda_{1}^{-2} \lambda_{2}^{-2}-3\right) / J_{\lim }^{\beta}}\right),
\end{aligned}
$$

where $\eta$ is the viscosity of the dashpot. So long as $\eta>0$, this kinetic model satisfies the thermodynamic inequality (12).

The viscoelastic relaxation time is defined by using the viscosity of the dashpot and the modulus of spring $\beta$,

$$
\tau_{V}=\eta / \mu^{\beta} .
$$

In reality, the elastomer may possess multiple relaxation times. This behavior may be incorporated in the model by introducing more parallel units of springs and dashpots.

Differentiating (19) and (20) with respect to time, and combining with (21) and (22), we obtain a set of four ordinary differential equations that evolve $\lambda_{1}(t), \lambda_{2}(t), \xi_{1}(t)$, and $\xi_{2}(t)$, once a program of loading is prescribed as functions $\sigma_{1}(t), \sigma_{2}(t)$, and $E(t)$.

We fit this viscoelastic model to uniaxial tensile tests conducted on VHB at several stretching rates. ${ }^{10}$ Fig. 5(a) shows good agreement between the model and the experiment. The experimental data obtained at three stretching rates can be fit to the model by using a common set of parameters: $\mu^{\alpha}=18 \mathrm{kPa}, \quad \mu^{\beta}=42 \mathrm{kPa}, \quad \tau_{V}=400 \mathrm{~s}, \quad J_{\lim }^{\alpha}=110 \quad$ and $J_{\lim }^{\beta}=55$. The shear moduli and relaxation time compare reasonably well with those reported elsewhere. ${ }^{10,20}$ The Gent model captures the strain-stiffening effect.

\section{MODEL OF LEAKAGE CURRENT}

Divide (1) by the change in time, and we write,

$$
i=\frac{d Q_{p}}{d t}+i_{\text {leak }}
$$

Here $i=d Q / d t$ is the current in the conducting wire, $d Q_{p} / d t$ is the rate of change of the polarizing charge accumulated on the electrodes, and $i_{\text {leak }}=d Q_{\text {leak }} / d t$ is the current leaking through the membrane. As illustrated in Fig. 3(b), the conservation of charge requires that current in the conducting wire should equal the sum of the rate of change of the polarizing charge on the electrodes and the current leaking through the membrane.

Electrical conduction in polymers is a complex phenomenon, which may depend on the molecular configuration, defects and impurities in the structure, and the type of electrodes, as well as temperature and moisture. ${ }^{23-25}$ Developing a model to capture these effects is beyond the scope of this paper. Instead, we propose a model based on following empirical observations. When the electric field is small, the leakage current is predominantly Ohmic and the leakage current density is approximately linear with the electric
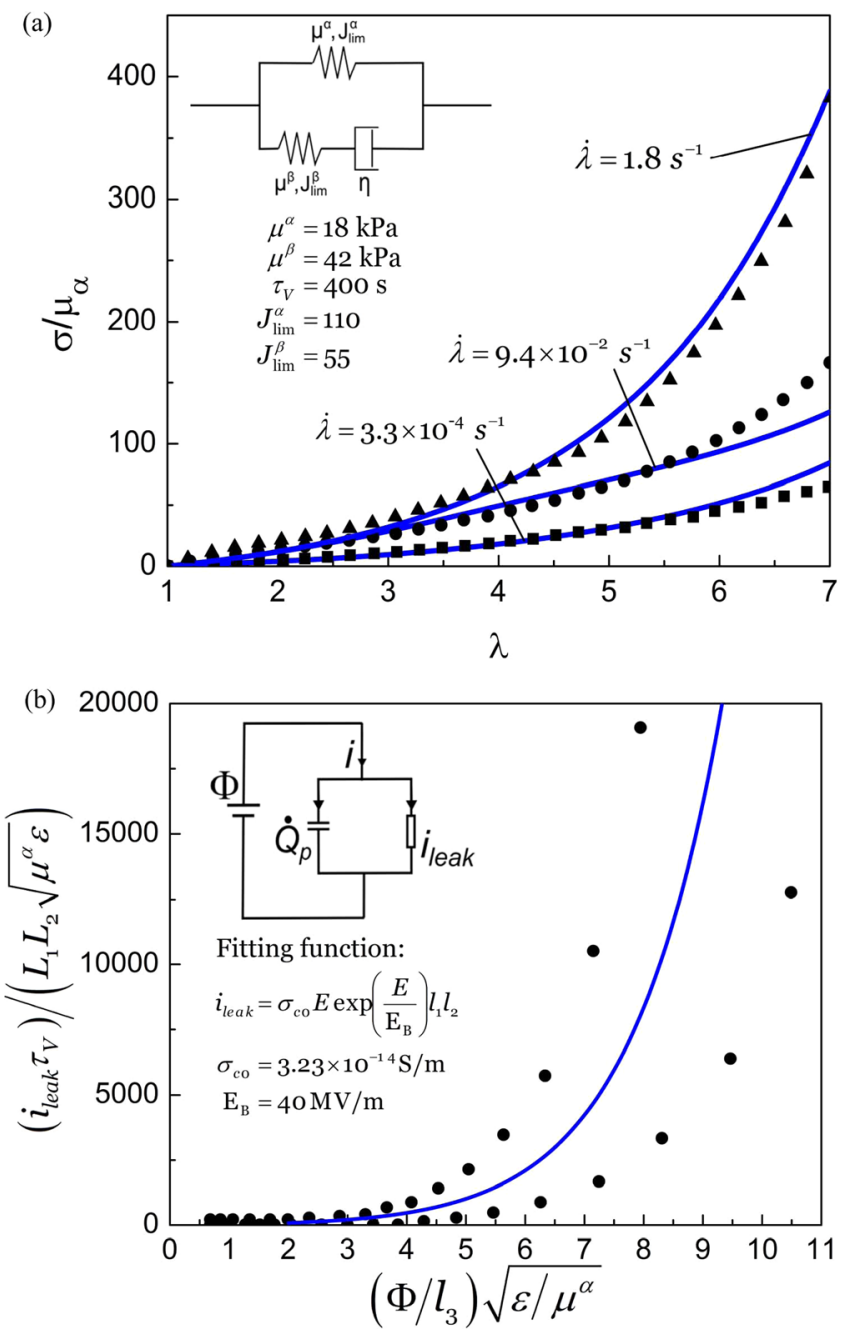

FIG. 5. (Color online) (a) Viscoelastic model is fitted to experimental data of Plante and Dubowsky. ${ }^{10}$ (b) Model of leakage current is fitted to experimental data of Gisby et al. ${ }^{17}$ 
field. ${ }^{24,26}$ As the electric field increases, the leakage current becomes non-Ohmic and increases exponentially. ${ }^{17,26}$ This non-Ohmic behavior becomes significant beyond a critical electric field, which is on the order of $10 \mathrm{MV} / \mathrm{m} .^{17,26,28}$ Recent experiments have reported that the conductivity for VHB 4910 increases exponentially under increasing electric field. $^{26}$

Define the density of the leakage current as $j_{\text {leak }}=i_{\text {leak }} /\left(l_{1} l_{2}\right)$. Based on the above observations, we relate the density of leakage current to the electric field as,

$$
j_{l e a k}=\sigma_{c}(E) E \text {. }
$$

The conductivity $\sigma_{c}(E)$ is taken to depend only on the electric field $E$ but not on deformation. So long as $\sigma_{c}(E)>0$, this kinetic model satisfies the thermodynamic inequality (3). We adopt the exponential function,

$$
\sigma_{c}(E)=\sigma_{0} \exp \left(\frac{E}{E_{B}}\right) .
$$

where $\sigma_{0}$ is the conductivity at low field, and $E_{B}$ is an empirical constant with the same dimension as the electric field. When $E \ll E_{B}$, the elastomer approximates an Ohmic conductor, that is, $j_{\text {leak }} \approx \sigma_{c 0} E$. As $E \rightarrow E_{B}$, the conductivity increases exponentially. ${ }^{26}$

The resistor-capacitor (RC) time constant is defined by the ratio between the permittivity and the conductivity of the dielectric,

$$
\tau_{R C}=\varepsilon / \sigma_{c 0}
$$

In Fig. 5(b), we fit the model of leakage current to experiments conducted on VHB 4905 actuators. ${ }^{17}$ The experiment

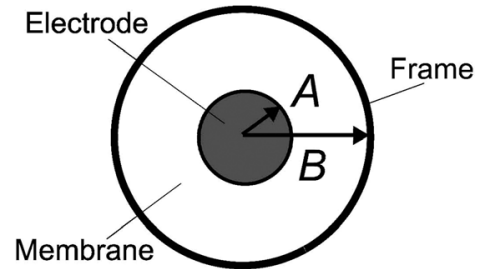

FIG. 6. Schematic of a circular actuator with electrodes coated on part of the surface of the elastomer.

confirms the exponential increase in leakage current at high electric fields. The fitting parameters are found to be $\sigma_{c 0}=3.23 \times 10^{-14} \mathrm{~S} / \mathrm{m}, E_{B}=40 \mathrm{MV} / \mathrm{m}$ and $\tau_{R C}=1230 \mathrm{~s}$. The permittivity of the membrane is taken to be $3.98 \times 10^{-11} \mathrm{~F} / \mathrm{m} .{ }^{10}$ Furthermore, the experimental data show significant scatter, especially at high fields. This indicates significant uncertainty in leakage current in the nonOhmic region. This uncertainty may be due to the varying quality of the films produced, ${ }^{17}$ which contributes to the complex physical nature of current leakage. We select two sets of experimental data that bounds the maximum- and minimum-observed leakage current over the range of measured electric fields. Our fitting shows qualitative agreement with the experimental data.

\section{ELECTROMECHANICAL BEHAVIOR OF DISSIPATIVE DIELECTRIC ELASTOMERS}

We now use the model of dissipative dielectric elastomers to simulate situations motivated by several recent experiments. ${ }^{14,26,40}$ These experiments were conducted by using the setup of circular actuators (Fig. 6). A membrane of a dielectric elastomer is pre-stretched and fixed to a rigid (a)

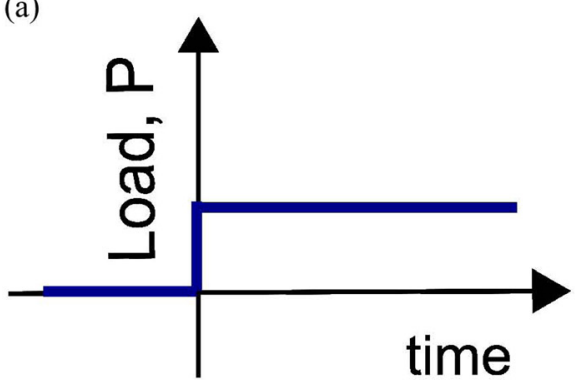

(b)

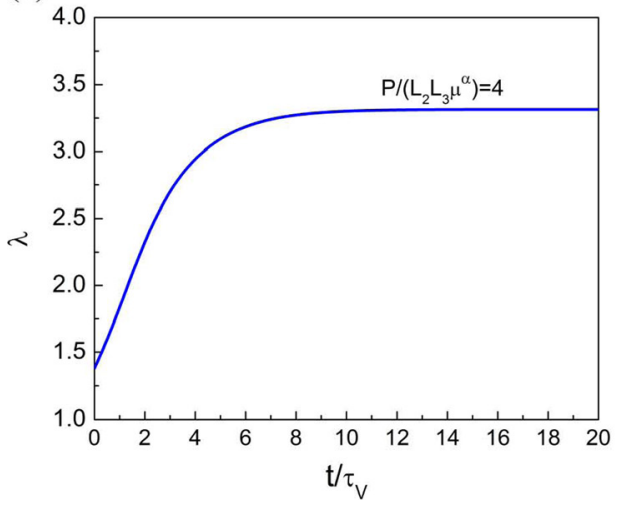

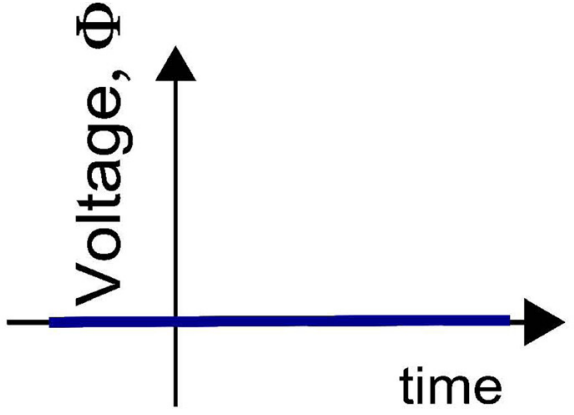

(c)

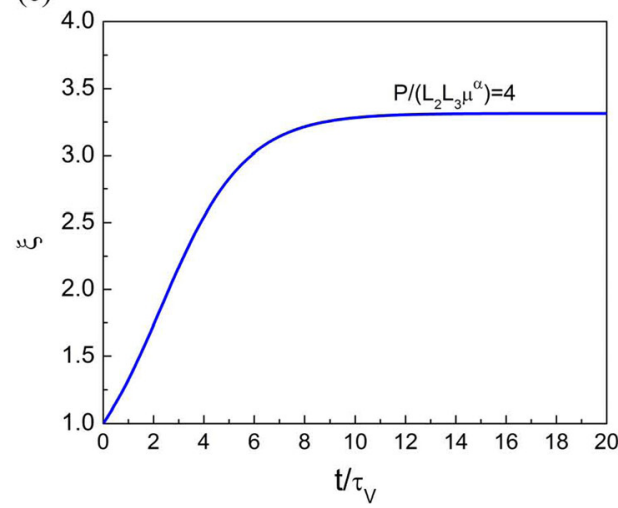

FIG. 7. (Color online) Under (a) constant mechanical load with no voltage, (b) the stretch of the membrane and (c) the stretch due to the dashpot, creep in time. 
frame. On part or on the entire surface of the membrane compliant electrodes are applied. The membrane is then left to relax for a long time. Subsequently, voltage or current is applied as a function of time. In our analyses, we assume that the membrane is subject to homogeneous, equal-biaxial deformation. Hence, $\lambda_{1}=\lambda_{2}=\lambda, \quad \xi_{1}=\xi_{2}=\xi \quad$ and $\sigma_{1}=\sigma_{2}=\sigma$. We focus on two dissipative mechanisms in our simulations-viscoelasticity and current leakage. We first model two experiments that study each effect in isolation, followed by experiments on actuation and voltage cycling.

To study the effect of viscoelastic relaxation in isolation, we model a creep experiment of a viscoelastic elastomer (Fig. 7). In this experiment, a force $P$ is applied at time $t=0$. Subsequently, the magnitude of the force is fixed, while the membrane creeps in time. In a short time, the dashpot does not deform, and the load is carried by both springs. In a long time, the dashpot deforms and the load is carried by the spring $\alpha$ alone. The characteristic time scale in this process is the viscoelastic relaxation time, $\tau_{V}$.
Next, we study the effect of current leakage in isolation. Our simulation was motivated by the experiment done by Rychkov et al. ${ }^{14}$ In this experiment, the setup is a circular actuator configuration with $A / B=1.0$ in Fig. 6 . Hence, the stretch remains fixed even when the voltage is applied. Keeping the stretch fixed prevent creep from taking place, allowing one to measure current leakage through the membrane as if it is a rigid capacitor. The membrane is first prestretched and fixed to a rigid frame (Fig. 8(a)). A long time was allowed to elapse for viscoelastic relaxation to take place. Upon equilibration, the membrane is charged up under a fixed voltage and held for a short time, before being switched to an open-circuit condition. Under the open-circuit condition, the current drawn from the voltage source $i$ drops to zero (Fig. 8(b)). Figures 8(c) and 8(d) show the timehistories of stress $\sigma$ and surface potential $\Phi$ across the membrane. $\sigma$ and $\Phi$ are measureable quantities, which may be corroborated with experimental results. We further plot the time-histories of stretch of the dashpot $\xi$ (Fig. 8(e)) and the polarizing charge on the electrodes $Q_{p}$ (Fig. 8(f)). These (a)

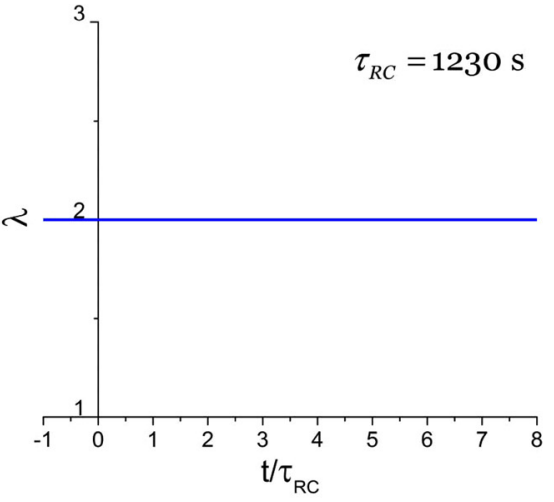

(c)

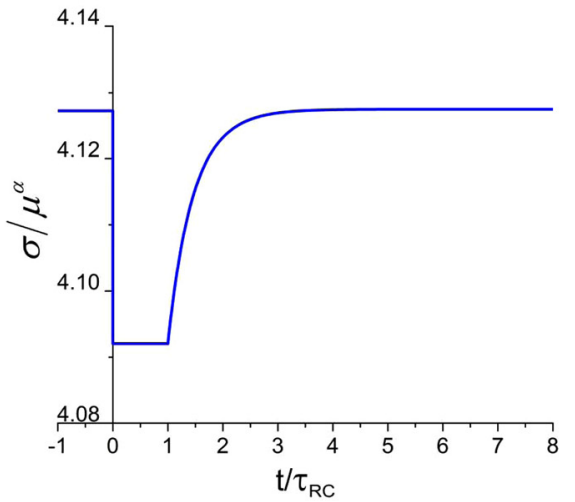

(e)

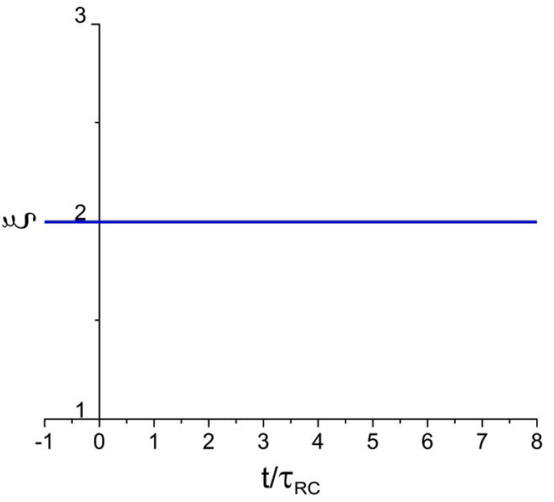

(b)

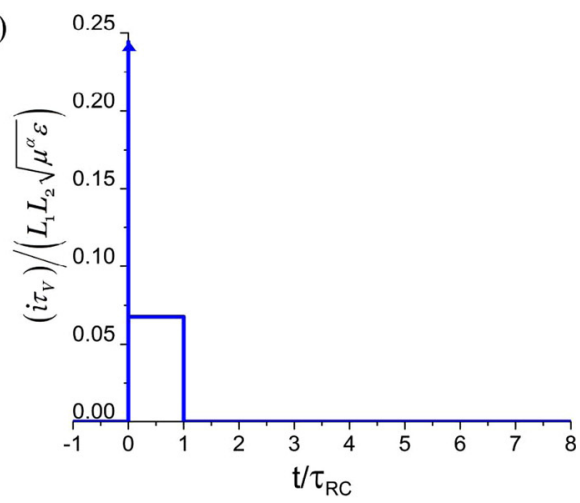

(d)

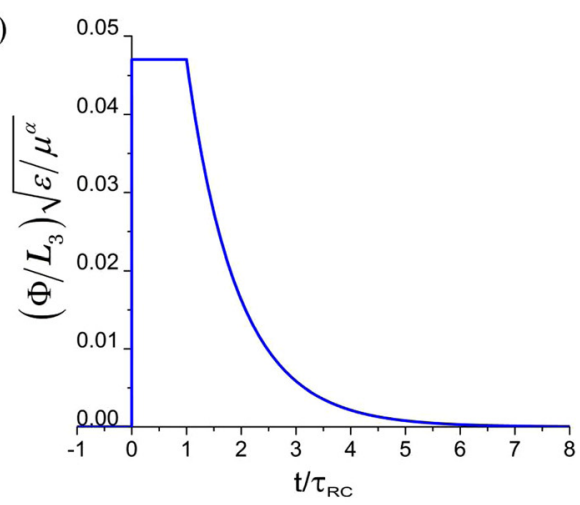

(f)

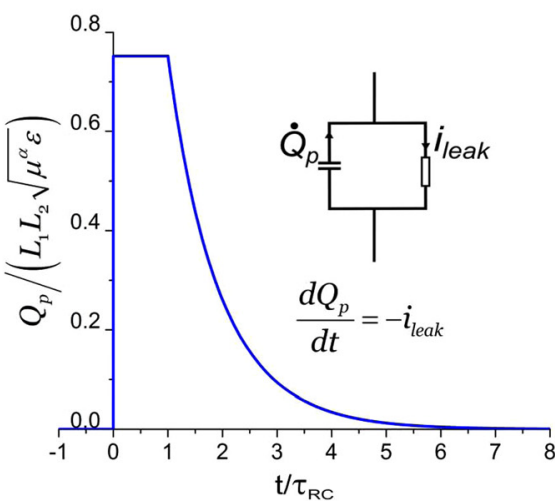

FIG. 8. (Color online) (a) A membrane is under a constant stretch. (b) The current in the conducting wire is suddenly switched on, held at a constant value for a period of charging with a fixed voltage, and then suddenly switched off. (c) stress, (d) voltage, (e) viscous stretch, and (f) charge on the electrodes. Charge leakage occurs through the membrane with a conductive relaxation time $\tau_{\mathrm{RC}}$ of $1230 \mathrm{~s}$. 
parameters provide important information on the respective dissipative processes of viscoelasticity and current leakage, but may not be directly measured in an experiment.

Figure 8(c) shows two features of stress response within a membrane. First, when a pre-stretched membrane is constrained from attaining further deformation, all viscoelastic effects like deformational creep and stress relaxation may be eliminated. This feature was manifested in the instantaneous drop in stress at the point of voltage application, and remained in the relaxed stress state when the voltage was maintained. The absence of viscoelastic effects was confirmed by Fig. 8(e), where the stretch in the dashpot remains constant over the entire test duration. Second, when the setup is switched to the open-circuit condition, charges on the electrodes will leak through the membrane. As more charges leak through the membrane, the stress in the membrane gradually increases. When all charges are leaked through, the stress returns to its original, uncharged level.

Our model may further be used as an aid to determine the optimal operating conditions of a dielectric elastomer generator. In such a generator, a membrane is first prestretched and pre-charged. Subsequent mechanical relaxation in the open-circuit condition provides a voltage boost across the electrodes. Current leakage in the open-circuit condition becomes an important process affecting its performance. ${ }^{41}$ If the relaxation process is too slow, leakage current will become very significant, resulting in a very small or even negative voltage boost, which adversely affects the conversion efficiency. This observation was confirmed by experiments in cycles operating at low frequencies. ${ }^{11}$ Our model may aid the design of cycle frequencies for generators that optimize the conversion efficiency.

Next, we analyze a similar setup as the previous example. In this experiment, all conditions remain the same as the previous one, with the exception of the input voltage. In the previous experiment, the voltage was stepped up instantaneously to a constant level, and maintained constant over a finite time. In this experiment, the voltage was ramped up from zero at a finite rate, up to a specific level, before being held constant (Fig. 9(b)). This was an experiment conducted (a)

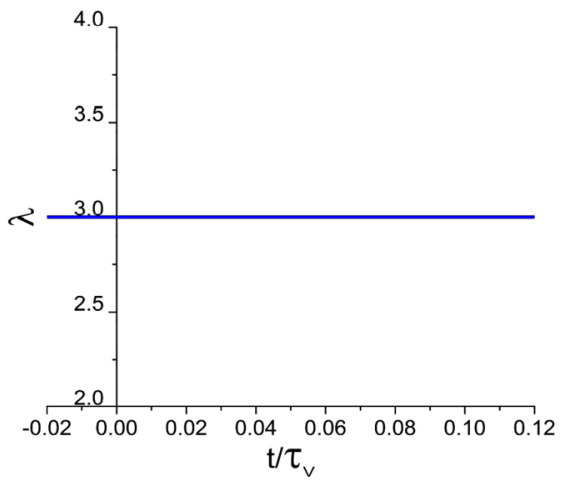

(c)

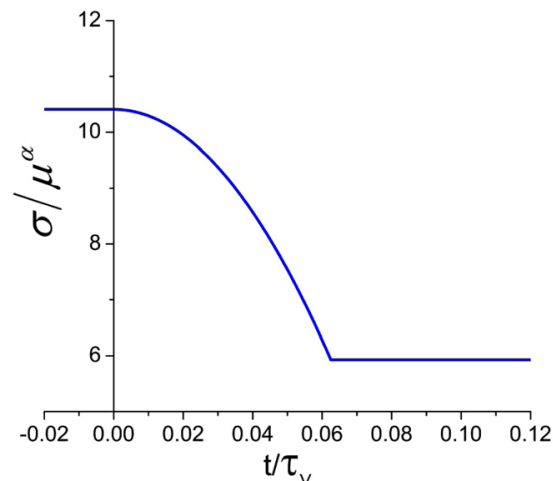

(e)

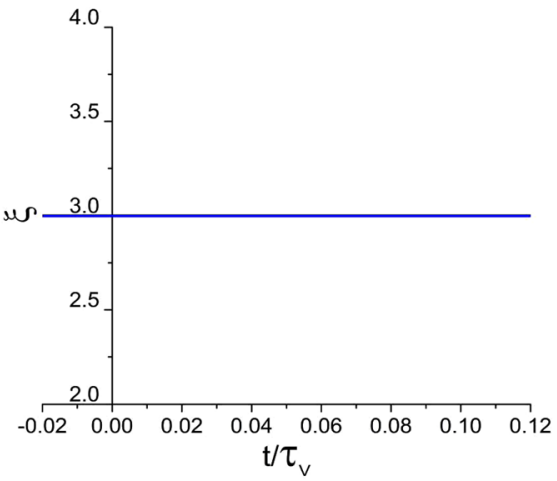

(b)

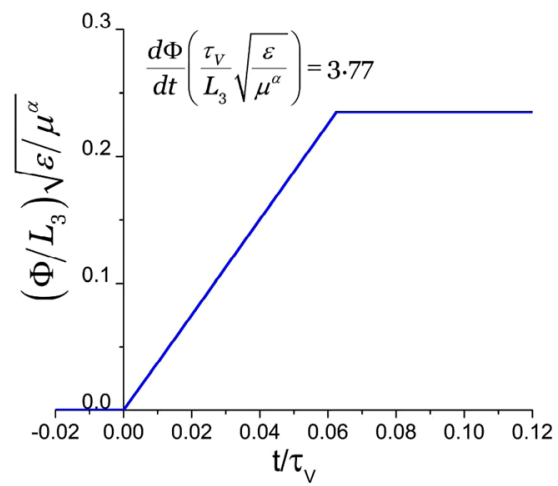

(d)

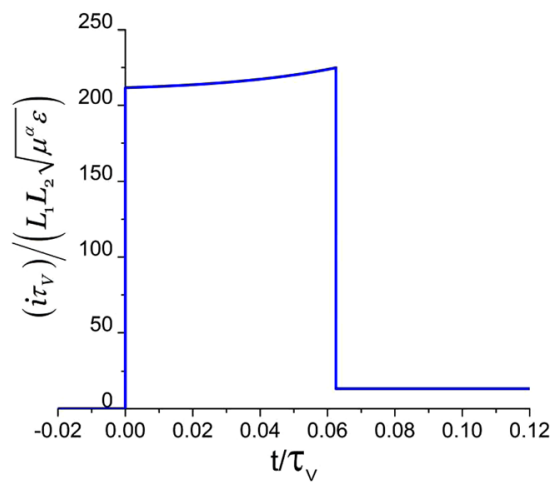

(f)

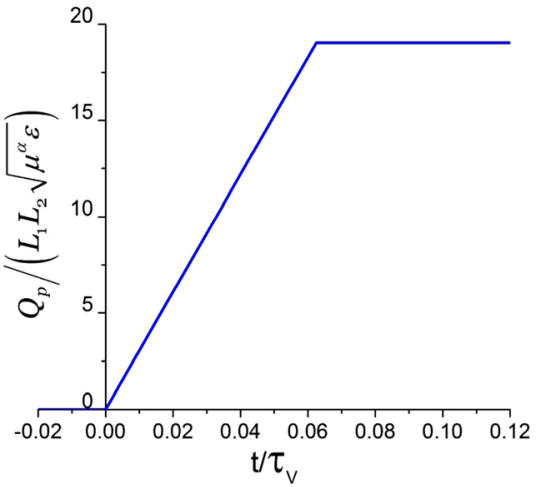

FIG. 9. (Color online) (a) A membrane is under a constant stretch. (b) Voltage is ramped up at a constant rate and is then held at a constant level. The calculated time-histories are shown for (c) stress, (d) current, (e) viscous stretch, and (f) charge on the electrodes. 
by Lillo et $a l^{26}$ The purpose of this experiment is to characterize the insulation and dielectric properties of acrylic dielectric elastomer actuators, and also allows one to plot the leakage current versus electric field of a DE. Because our current leakage model is fitted to experimental data, ${ }^{17}$ the leakage current versus electric field plot would be identical to that in Fig. 5(b). Similar to the previous example, viscoelastic effects were eliminated (Fig. 9(e)). Both the current $i$ and charge on the electrodes $Q_{p}$ increase initially as the voltage increases (Figs. 9(d) and 9(f)). The charge on the electrodes is given by

$$
Q_{p}=C \Phi,
$$

where $C$ is the capacitance of the membrane, given as $C=\varepsilon l_{1} l_{2} / l_{3}$. A combination of (24) and (28) gives that

$$
i=\Phi \frac{d C}{d t}+C \frac{d \Phi}{d t}+i_{\text {leak }} .
$$

The total current is contributed by three processes-deformation change $(d C / d t)$, voltage change $(d \Phi / d t)$ and leakage current $\left(i_{\text {leak }}\right)$. In this experiment, the deformation is constrained by the rigid frame, hence $d C / d t=0$. The total cur-

(a)
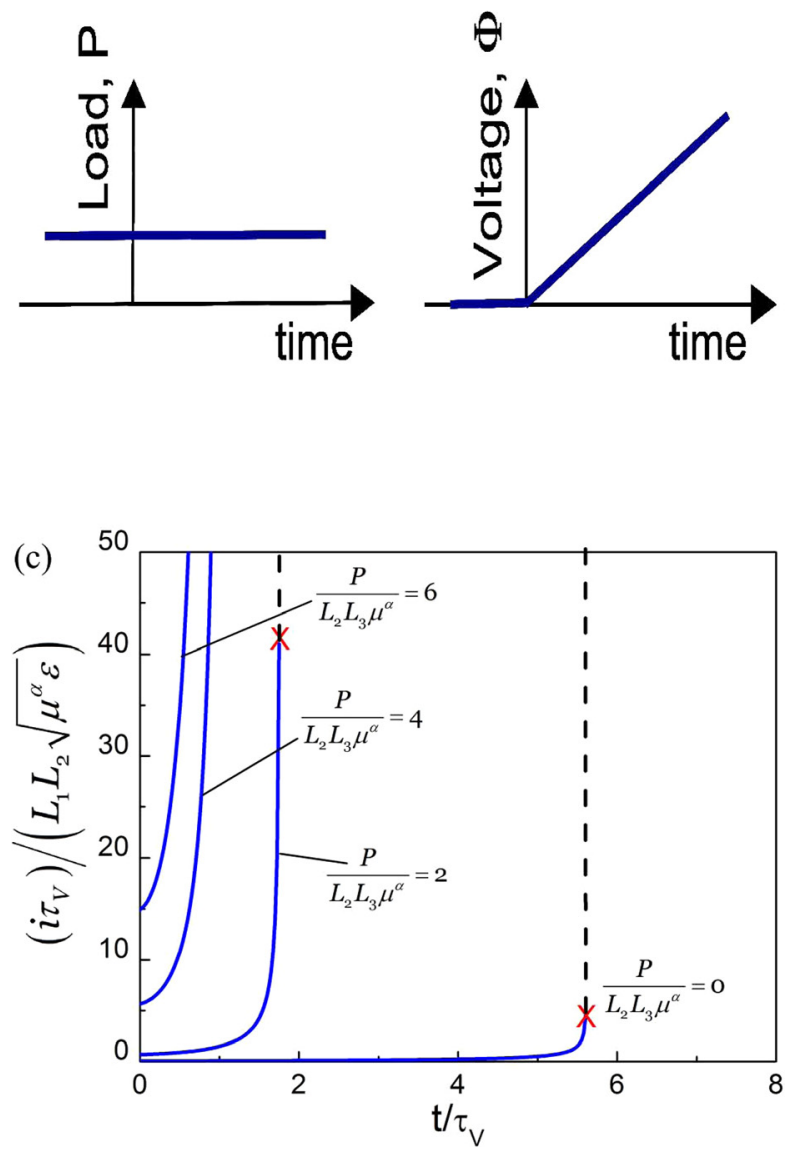

rent is therefore contributed by voltage change $(d \Phi / d t)$ and current leakage. Figures 9(b) and 9(d) clearly show that $i_{\text {leak }}>0$. At higher electric fields, Fig. 9(d) shows an exponential increase in $i_{\text {leak }}$, which is in qualitative agreement with experiments. ${ }^{17,26}$ Because $d C / d t=0$, Figs. 9(b) and 9(f) show that the charges on the electrodes increase linearly with voltage. Once the voltage is held constant, $d \Phi / d t=0$, any contribution to total current is due to the leakage current. The leakage current provides a measure of the conductive behavior of the actuator subject to high electric fields. The model allows one to calculate the leakage current and assess the electrical response of the actuator under conditions of high electric field, which is essential to achieve large electrical actuation. Our model may therefore be used to aid the design of efficient actuation cycles.

It is known that pre-stretching a dielectric elastomer may significantly enhance its actuation performance. ${ }^{1,10,38,42}$ When the radius of the electrodes is much smaller than that of the membrane, $A \ll B$, we simplify the setup in the simulation by assuming the active part of the membrane is subject to a constant force. In this example, we study the effect of pre-stress on the current consumption of an actuator. Figure 10(a) illustrates the loading program: a voltage ramps up
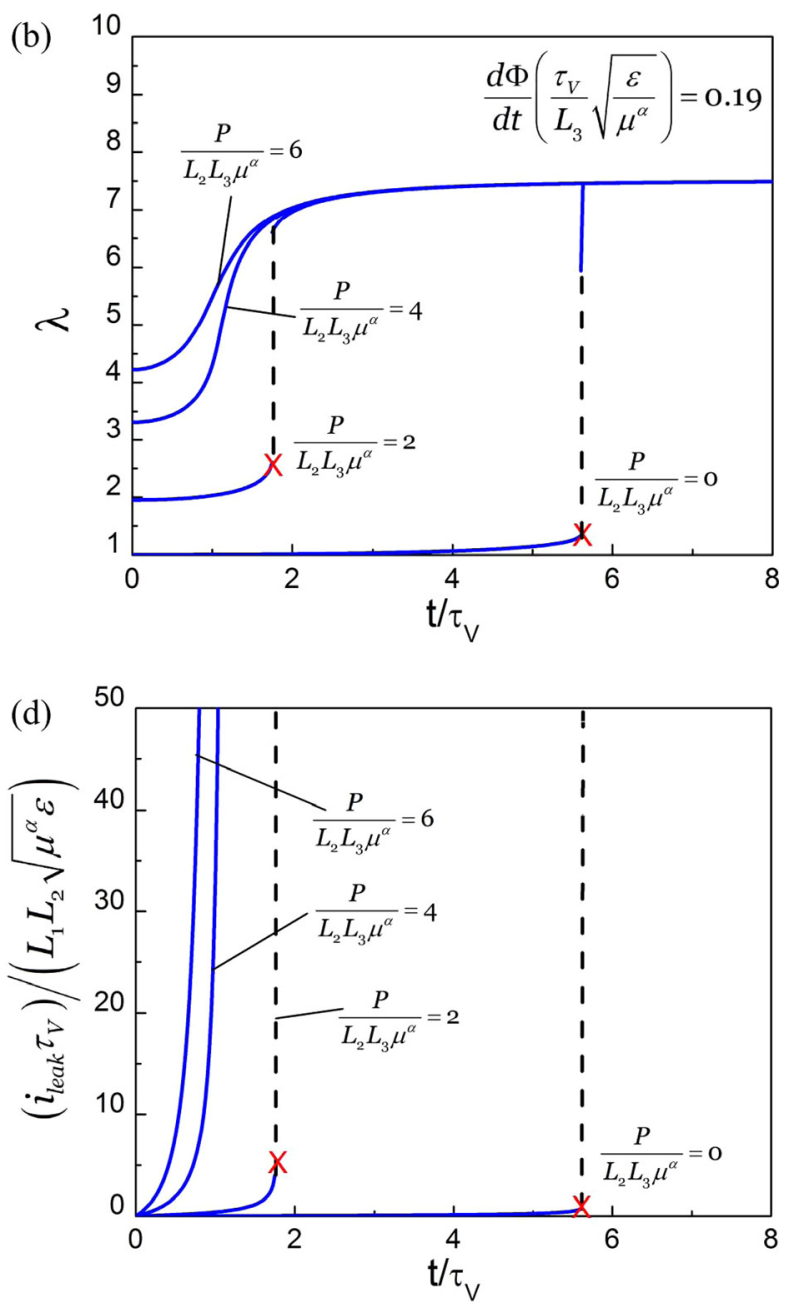

FIG. 10. (Color online) (a) A membrane is subject to a constant force and a ramping voltage. The calculated time-histories are shown for (b) the stretch of the membrane, (c) the current in the conducting wire, and (d) the leakage current through the membrane. At a low level of the applied force, a snap-through instability occurs, marked by a red cross $\mathrm{X}$ and a vertical dashed line. 
with time under a constant pre-stress. We consider a constant ramping voltage rate for all cases in Fig. 10. As voltage is increased, the stretch of the dielectric elastomer increases (Fig. 10(b)). At zero or low pre-stresses, the stretch increases up to a point denoted by a red cross, $\mathrm{X}$. At this point, electromechanical instability (EMI) occurs. ${ }^{42-44}$ As the voltage is increased, the electric field increases with the expanding elastomer. At some point, the same voltage will induce an even higher field; a positive feedback develops between a thinning dielectric elastomer and an increasing electric field-EMI occurs. EMI causes an instantaneous jump from a low stretch level to a high stretch level (Fig. 10(b)), usually leading to electrical breakdown. It is now well known that EMI limits the actuation of a dielectric elastomer to $<50 \%$ actuation strain, ${ }^{45,46}$ and that a dielectric elastomer may be stabilized against EMI by pre-stretch or pre-stress, leading to large actuation. 1,10,35,42 Analytically, EMI occurs when the determinant of the Hessian of the free energy function prescribed in Eq. (14) vanishes. ${ }^{20,45}$ An explicit form of the Hessian can be found in a previous paper. ${ }^{45}$ Fig. 10(b) shows that, at zero or low pre-stress, the membrane undergoes "snap-through" deformation ${ }^{33}$ after EMI occurs. When prestress is increased, EMI is eliminated, allowing the dielectric elastomer to attain large actuation. Consequently, large actuation also implies very high electric field; leading to an exponential increase in leakage current (Fig. 10(d)) and, hence, huge current consumption (Fig. 10(c)). Although large actuation performance may be achieved at higher prestress, the efficiency of such a cycle may be low due to substantial leakage current losses. This observation is consistent (a)

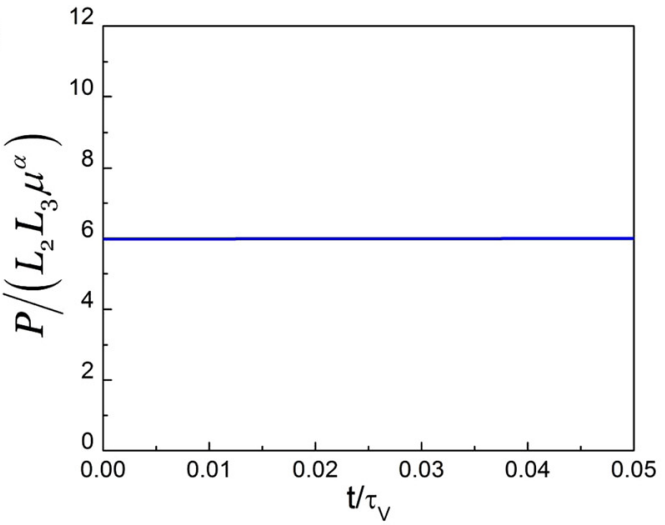

(c)

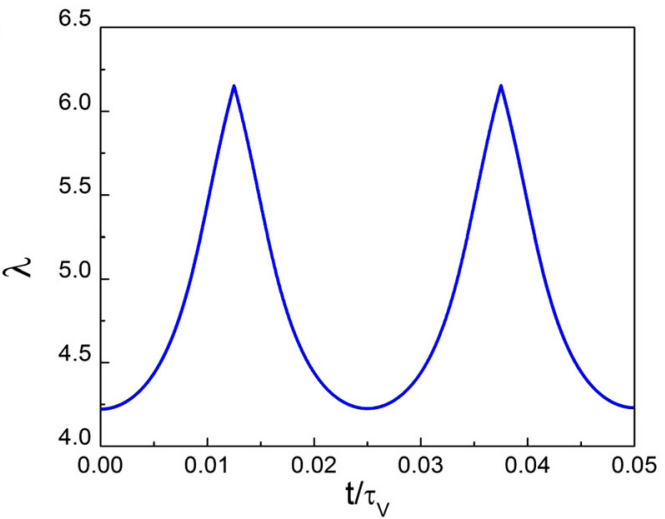

(e)

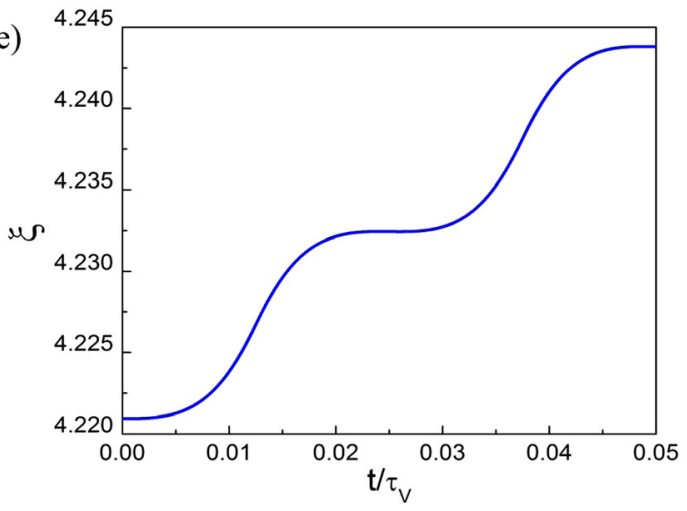

(b)

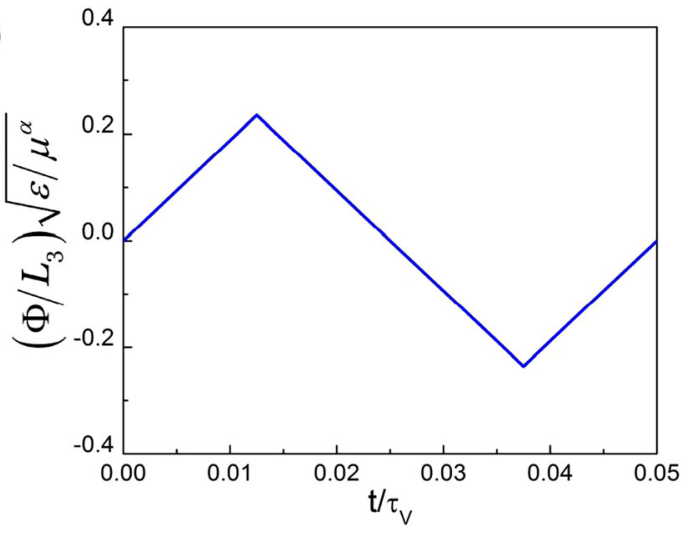

(d)
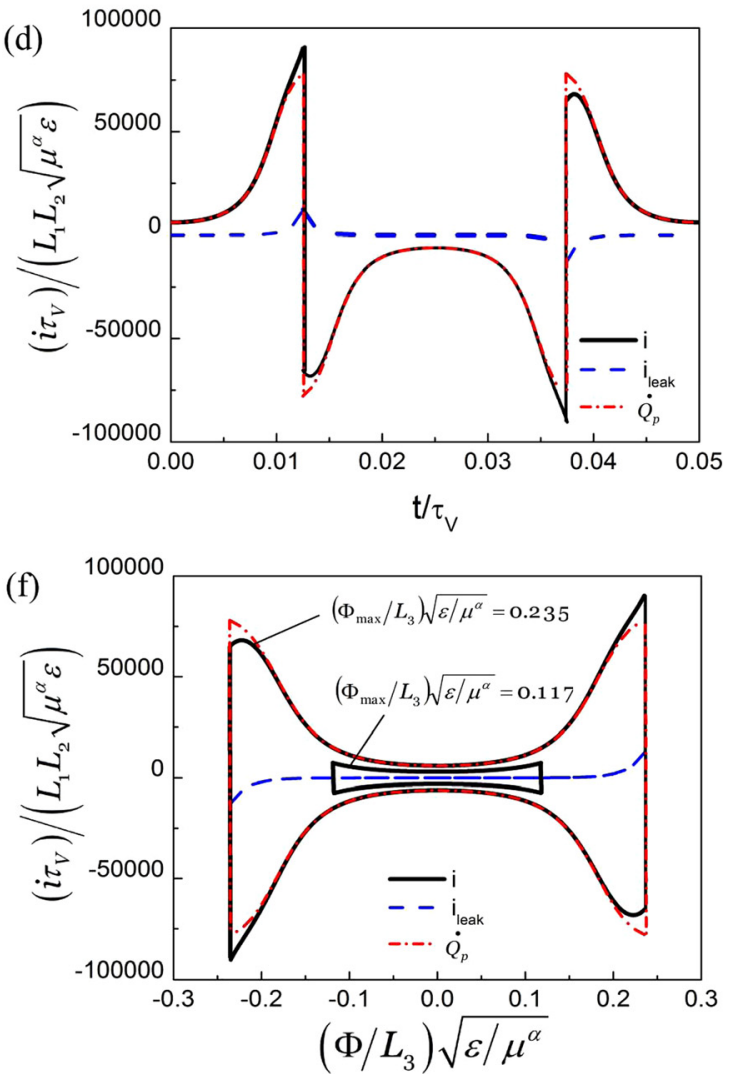

FIG. 11. (Color online) A membrane is subject to (a) a constant force and (b) cyclic voltage. As a result of the fast loading, the viscous loss is small, as indicated by the total and viscous stretches in (c) and (e), respectively. When current leakage becomes significant (d), the current-voltage response in (f) is not symmetric, indicating hysteresis. 
with experimental observations, which show current leakage losses reduce the efficiency of actuation cycles to $1 \%$ under large actuation stretches. ${ }^{11}$ To optimize conversion efficiency, one may then need to consider a compromise between large actuation and large current leakage losses.

As a final example, we analyze the response of an actuator subject to a cyclic electric loading (Fig. 11). Such periodic loading will most closely resemble actual operating conditions of an actuator. This set of calculations follows the experiment reported by Bauer and Paajanen. ${ }^{40}$ In that experiment, a circular dot actuator was used (as shown in the schematic in Fig. 6). In our simulations, we shall approximate the boundary conditions for the active dielectric elastomer membrane as being held under a constant pre-stress (Fig. 11(a)), and subject to a triangular cyclic voltage (Fig. 11(b)). Such approximation may not exactly replicate the actual physical conditions in the experiment, but provides a qualitative analysis of the setup, especially when B $\gg$ A in Fig. 6. For exact analysis of this problem, a boundary-value-problem must be solved. The solution to this problem was presented in a separate paper, ${ }^{42}$ and shall not be repeated here.

In this experiment, the voltage was varied on a timescale much faster than the viscoelastic relaxation time (Fig. 11(b)). As such, the actuation is relatively large (Fig. 11(c)), whereas the viscous effect is negligible due to the fast loading rate (Fig. 11(e)). On the other hand, due to the large actuation and, hence, electric field, current leakage is significant, which makes up about $10 \%$ of the total current $i$ at its maximum (Fig. 11(d)). This produces an asymmetric current-voltage response, as observed in the experiment. ${ }^{40}$ For a rigid capacitor, the current-voltage response is rectangular in shape. In Fig. 11(f), a current-voltage response for the dielectric elastomer subject to a smaller voltage amplitude is included for comparison. At that low voltage level, the current-voltage response is almost similar to that of a rigid capacitor. Any deviation from the rectangular shaped current-voltage response is due to the change in capacitance of the DE, the voltage change, and current leakage losses (Eq. (29)). Given that dielectric elastomer actuators are primarily driven by electric fields, current may be an important parameter for actuator control. ${ }^{16,40}$ Current-voltage measurements may then provide useful information about the actuation and leakage losses, ${ }^{40}$ and such measurements may be complemented by our model, as illustrated here.

\section{CONCLUSION}

We use nonequilibrium thermodynamics to construct a model of dissipative dielectric elastomers. We focus on two dissipative processes-viscoelasticity and current leakage. This theory is then applied to study the electromechanical response of a membrane subject to time-dependent loadings. Simple descriptions of viscoelasticity and current leakage are used to predict dynamic responses of the membrane. The method may be adapted to analyze more sophisticated modes of deformation and material models. The main results may be summarized as follows. Large actuation strains may be achieved for a pre-stressed actuator, but this is done at the expense of greater losses due to current leakage. One may need to find a compromise between large actuation strains and good conversion efficiency. When operating as a generator at low frequencies, current leakage may adversely affect the ability of the membrane to store charges in an opencircuit condition, leading to a very poor voltage boost. We also find that current leakage significantly modifies the current-voltage characteristics of dielectric elastomers under cyclic electric fields with high amplitudes. It is hoped that the method may be used as an aid to optimize the conversion efficiency of membrane actuation and generation cycles.

\section{ACKNOWLEDGMENTS}

The work at Harvard is supported by the U.S. Army Research Office (ARO) (W911NF-09-1-0476), Defense Advanced Research Projects Agency (DARPA) (W911NF10-1-0113), and Materials Reseach Science and Engineering Center (MRSEC). C. C. Foo acknowledges the Post-doctoral Fellowship awarded by the Agency for Science, Technology and Research (A*STAR), Singapore. S. J. A. Koh acknowledges the start-up funding from Ministry of Education (Singapore), administered through the National University of Singapore. S. Bauer acknowledges the support by the Austrian Science Fund (FWF-P22912-N20).

\section{APPENDIX: A MODEL OF DISSIPATIVE DIELECTRIC ELASTOMERS}

The model described in Sec. II is written here in a general form for inhomogeneous fields in three dimensions. A review of related models can be found in a previous work. ${ }^{33}$ Consider a body of an elastomer in three dimensions. The elastomer in the undeformed state is chosen as the reference state, where each material particle is labeled by its coordinate $\mathbf{X}$. In the deformed state at time $t$, the particle $\mathbf{X}$ moves to a place with coordinate $\mathbf{x}(\mathbf{X}, \mathrm{t})$. The deformation gradient is defined by,

$$
F_{i K}=\frac{\partial x_{i}(\mathrm{X}, t)}{\partial X_{K}}
$$

Imagine that every material particle $\mathbf{X}$ is attached with a battery of electric potential $\Phi(\mathbf{X})$. Define the nominal electric field by,

$$
\tilde{E}_{K}=-\frac{\partial \Phi(\mathbf{X}, t)}{\partial X_{K}} .
$$

When the dielectric is in the reference state, denote an element of volume by $d V(\mathbf{X})$. On each element of volume, we prescribe force $\mathbf{B}(\mathbf{X}, t) d V(\mathbf{X})$. In the reference state, denote an element area of the surface of the dielectric by $d A(\mathbf{X})$, and denote the unit vector normal to the element by $\mathbf{N}$, pointing outside the dielectric. Parts of the surface of the dielectric may be prescribed with displacement, and other parts of the surface of the dielectric may be prescribed with forces. For example, on an element of the surface of the dielectric, $d A(\mathbf{X})$, we may prescribe force $\mathbf{T}(\mathbf{X}) d A(\mathbf{X})$, where $\mathbf{T}$ is the nominal density of the surface force (i.e., the traction). Force balance requires that the nominal stress $s_{i K}$ satisfy that, 


$$
\frac{\partial s_{i K}}{\partial X_{K}}+B_{i}=0
$$

in the volume, and that,

$$
\left(s_{i K}^{-}(\mathbf{X}, t)-s_{i K}^{+}(\mathbf{X}, t)\right) N_{K}(\mathbf{X}, t)=T_{i}(\mathbf{X}, t),
$$

on an interface.

Similarly, denote $\tilde{q}(\mathrm{X}, t) d V(\mathrm{X})$ to be the charge in an element of volume, and $\tilde{\omega}(\mathrm{X}, t) d A(\mathrm{X})$ the charge on an element of an interface. The nominal electric displacement obeys that,

$$
\frac{\partial \tilde{D}_{K}}{\partial X_{K}}=\tilde{q}
$$

in the volume of the body, and,

$$
\left(\tilde{D}_{K}^{+}(\mathrm{X}, t)-\tilde{D}_{K}^{-}(\mathrm{X}, t)\right) N_{K}(\mathrm{X}, t)=\tilde{\omega}(\mathrm{X}, t),
$$

on the surface of the body.

In the deformed state, let the charges per unit time pumped into a volume element be $r(\mathrm{X}, t) d V(\mathrm{X})$, and into an interface element be $i(\mathrm{X}, t) d A(\mathrm{X})$. The charge also migrates in the dielectric. In the deformed state, let $J_{K}(\mathrm{X}, t) N_{K} d A(\mathrm{X})$ be the charge per unit time crossing an element of area. The conservation of charge requires that,

$$
\frac{\partial \tilde{q}}{\partial t}+\frac{\partial J_{K}}{\partial X_{K}}=r
$$

in the volume, and,

$$
\frac{\partial \tilde{\omega}}{\partial t}+\left(J_{K}^{+}(\mathbf{X}, t)-J_{K}^{-}(\mathbf{X}, t)\right) N_{K}(\mathbf{X}, t)=i(\mathbf{X}, t),
$$

on an interface.

Let $W d V(\mathbf{X})$ be the Helmholtz free energy associated with an element of volume. Thermodynamics requires that the increase in the free energy of the dielectric should not exceed the total work done, namely,

$$
\int \frac{\delta W}{\delta t} d V \leq \int \Phi r d V+\int \Phi i d A+\int B_{i} \frac{\partial x_{i}}{\partial t} d V+\int T_{i} \frac{\partial x_{i}}{\partial t} d A
$$

This inequality holds for every possible dissipative process. A combination of the above equations gives that,

$$
\delta \dot{W} \leq s_{i K} \delta \dot{F}_{i K}+\tilde{E}_{K} \delta \dot{\tilde{D}}_{K}+\tilde{E}_{K} J_{K}
$$

As a material model, the density of the Helmholtz free energy is taken to be a function of a set of independent variables,

$$
W=W\left(\mathbf{F}, \tilde{\mathbf{D}}, \xi_{\alpha}, \xi_{\beta}, \ldots\right),
$$

where $\xi_{\alpha}, \xi_{\beta}, \ldots$ are internal variables that measure the progress of relaxation processes. Inserting equation (11) into (10), following similar assumptions as in Sec. II, we obtain that,

$$
\begin{gathered}
s_{i K}=\frac{\partial W\left(\mathbf{F}, \tilde{\mathbf{D}}, \xi_{\alpha}, \xi_{\beta}, \ldots\right)}{\partial F_{i K}}, \\
\tilde{E}_{K}=\frac{\partial W\left(\mathbf{F}, \tilde{\mathbf{D}}, \xi_{\alpha}, \xi_{\beta}, \ldots\right)}{\partial \tilde{D}_{K}}, \\
\sum_{\gamma} \frac{\partial W\left(\mathbf{F}, \tilde{\mathbf{D}}, \xi_{\alpha}, \xi_{\beta}, \ldots\right)}{\partial \xi_{\gamma}} \delta \xi_{\gamma} \leq 0, \\
\tilde{E}_{K} J_{K} \geq 0 .
\end{gathered}
$$

${ }^{1}$ R. Pelrine, R. Kornbluh, Q. Pei, and J. Joseph, Science 287, 836 (2000).

${ }^{2}$ F. Carpi, S. Bauer, and D. De Rossi, Science 330, 1759 (2010).

${ }^{3}$ N. Galler, H. Ditlbacher, B. Steinberger, A. Hohenau, M. Dansachmüller, F. Camacho-Gonzales, S. Bauer, J. R. Krenn, A. Leitner, and F. R. Aussenegg, Appl. Phys. B 85, 7 (2006).

${ }^{4}$ G. Kofod, M. Paajanen, and S. Bauer, Appl. Phys. A 85, 141 (2006).

${ }^{5}$ G. Kovacs, L. Düring, S. Michel, and G. Terrasi, Sens. Actuators A 155 , 299 (2009).

${ }^{6}$ F. Carpi, G. Frediani, M. Nanni, and D. D. Rossi, IEEE/ASME Trans. Mechatron. 16, 16 (2011).

${ }^{7}$ C. Keplinger, M. Kaltenbrunner, N. Arnold, and S. Bauer, Proc. Natl. Acad. Sci. U.S.A. 107, 4505 (2010).

${ }^{8}$ F. Carpi, D. D. Rossi, R. Kornbluh, R. Pelrine, and P. Sommer-Larsen, Dielectric Elastomers as Electromechanical Transducers, 1st ed. (Elsevier, Amsterdam, 2008).

${ }^{9}$ T. McKay, B. O’Brien, E. Calius, and I. Anderson, Smart Mater. Struct. 19, 055025 (2010).

${ }^{10}$ J. S. Plante and S. Dubowsky, Int. J. Solids Struct. 43, 7727 (2006).

${ }^{11}$ J. S. Plante and S. Dubowsky, Sens. Actuators A 137, 96 (2007).

${ }^{12}$ M. Molberg, Y. Leterrier, C. J. G. Plummer, C. Walder, C. Löwe, D. M. Opris, F. A. Nüesch, S. Bauer, and J. -A. E.E. Månson, J. Appl. Phys. 106, 054112 (2009).

${ }^{13}$ A. York, J. Dunn, and S. Seelecke, Smart Mater. Struct. 19, 094014 (2010).

${ }^{14}$ D. Rychkov, M. Dansachmuller, H. Ragusch, A. Becker, and G. Kofod, in 2010 10th IEEE International Conference on Solid Dielectrics (Potsdam, Germany, 2010), pp. 1.

${ }^{15}$ P. Lochmatter, G. Kovacs, and M. Wissler, Smart Mater. Struct. 16, 477 (2007).

${ }^{16}$ C. Keplinger, M. Kaltenbrunner, N. Arnold, and S. Bauer, Appl. Phys. Lett. 92, 192903 (2008).

${ }^{17}$ T. A. Gisby, S. Q. Xie, E. P. Calius, and I. A. Anderson, Proc. SPIE 7642, 764213 (2010).

${ }^{18}$ G. Kofod and P. Sommer-Larsen, in Dielectric Elastomers as Electromechanical Transducers, edited by F. Carpi, D. D. Rossi, R. Kornbluh, R. E. Pelrine, and P. Sommer-Larsen (Elsevier, Amsterdam, 2008), pp. 159.

${ }^{19} \mathrm{M}$. Wissler and E. Mazza, in Dielectric Elastomers as Electromechanical Transducers, edited by F. Carpi, D. D. Rossi, R. Kornbluh, R. E. Pelrine, and P. Sommer-Larsen (Elsevier, Amsterdam, 2008), pp. 169.

${ }^{20}$ X. Zhao, S. J. A. Koh, and Z. Suo, Int. J. Appl. Mech. 3, 1 (2011).

${ }^{21}$ A. S. A. Reffaee, D. E. El Nashar, S. L. Abd-El-Messieh, and K. N. Abd-El Nour, Mater. Des. 30, 3760 (2009).

${ }^{22}$ M. Seki and K. Sato, Macromol. Chem. Phys. 196, 1813 (1995).

${ }^{23}$ D. A. Seanor, Adv. Polym. Sci. 4, 317 (1965).

${ }^{24}$ G. G. Raju, in Dielectrics in Electric Fields (Marcel Dekker, New York, 2003), pp. 329-382.

${ }^{25}$ A. R. Blythe and D. Bloor, in Electrical Properties of Polymers (Cambridge University Press, Cambridge, U.K., 2005), pp. 111-153.

${ }^{26}$ L. Di Lillo, A. Schmidt, A. Bergamini, P. Ermanni, and E. Mazza, Proc. SPIE 7976, 79763B (2011).

${ }^{27}$ C.-C. Chiang, I.-H. Ko, M.-C. Chen, Z.-C. Wu, Y.-C. Lu, S.-M. Jang, and M.-S. Liang, J. Electrochem. Soc. 151, 93 (2004).

${ }^{28}$ J.-P. Crine, IEEE T. Dielect. El. In. 4, 487-495 (1997).

${ }^{29}$ C. Dang, J.-L. Parpal, and J.-P. Crine, IEEE T. Dielect. El. In. 3, 237-247 (1996).

${ }^{30}$ J.-L. Parpal, J.-P. Crine, and C. Dang, IEEE T. Dielect. El. In. 4, 197-209 (1997).

${ }^{31}$ J.-P. Crine, IEEE Trans. Dielectr. Electr. Insul. 9, 697 (2002). 
${ }^{32}$ N. Parkman, in Physics of Plastics, edited by P. D. Ritchie (Iliffe, London, 1965), pp. 285-322.

${ }^{33}$ Z. Suo, Acta Mech. Solida Sinica 23, 549 (2010).

${ }^{34}$ D. I. Bower, in An Introduction to Polymer Physics (Cambridge University Press, Cambridge, U.K., 2002), pp. 248-283.

${ }^{35}$ G. Kofod, P. Sommer-Larsen, R. Kornbluh, and R. Pelrine, J. Intell. Mater. Syst. Struct. 14, 787 (2003).

${ }^{36}$ X. Zhao, W. Hong, and Z. Suo, Phys. Rev. B 76, 134113 (2007).

${ }^{37}$ J. S. Bergstrom and M. C. Boyce, J. Mech. Phys. Solids 46, 931 (1998).

${ }^{38}$ W. Hong, J. Mech. Phys. Solids 59, 637 (2011).
${ }^{39}$ A. N. Gent, Rubber Chem. Tech. 69, 59 (1996).

${ }^{40}$ S. Bauer and M. Paajanen, Proc. SPIE 6168, 61682K (2006).

${ }^{41}$ S. J. A. Koh, X. Zhao, and Z. Suo, Appl. Phys. Lett. 94, 262902 (2009).

${ }^{42}$ S. J. A. Koh, T. Li, J. Zhou, X. Zhao, W. Hong, J. Zhu, and Z. Suo, J. Polym. Sci. Part B: Polym. Phys. 49, 504 (2011).

${ }^{43}$ Z. Suo and J. Zhu, Appl. Phys. Lett. 95, 232909 (2009).

${ }^{44}$ X. Zhao and Z. Suo, Phys. Rev. Lett. 104, 178302 (2010).

${ }^{45}$ X. Zhao and Z. Suo, Appl. Phys. Lett. 91, 061921 (2007).

${ }^{46}$ R. Pelrine, R. Kornbluh, Q. Pei, and J. Joseph, Science 287, 836 (2000). 